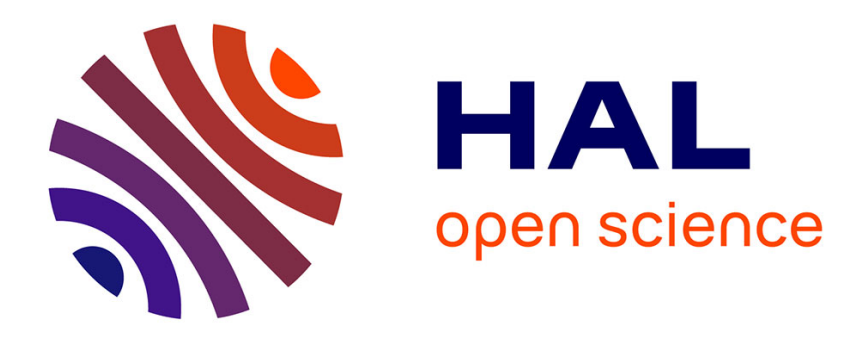

\title{
Bottleneck models and departure time problems
}

André de Palma, Claude Lefèvre

\section{To cite this version:}

André de Palma, Claude Lefèvre. Bottleneck models and departure time problems. 2018. hal01581519v2

\section{HAL Id: hal-01581519 \\ https://hal.science/hal-01581519v2}

Preprint submitted on 5 Jan 2018

HAL is a multi-disciplinary open access archive for the deposit and dissemination of scientific research documents, whether they are published or not. The documents may come from teaching and research institutions in France or abroad, or from public or private research centers.
L'archive ouverte pluridisciplinaire HAL, est destinée au dépôt et à la diffusion de documents scientifiques de niveau recherche, publiés ou non, émanant des établissements d'enseignement et de recherche français ou étrangers, des laboratoires publics ou privés. 


\title{
Bottleneck models and departure time problems
}

\author{
André de Palma, Ecole Normale Supérieure (ENS) Paris-Saclay ${ }^{1}$ \\ Claude Lefèvre, Université Libre de Bruxelles (ULB) ${ }^{2}$
}

January 6, 2018

\begin{abstract}
This paper is concerned with the analysis of departure times in dynamic bottleneck models. First, the case of a set of individual drivers is discussed through both deterministic and stochastic approaches. Then, the analysis is extended to a new model that combines small and large agents. In the stochastic setting, our focus is mainly on the model building; simulations will be carried out in a near future.
\end{abstract}

Keywords: dynamic bottleneck models; congestion; equilibrium; deterministic version; stochastic approach; small and large agents; discrete choice models.

\section{Introduction}

Dynamic bottleneck models have become very popular in Transportation. This has not always been the case. In the early eighties, most transportation models were static, in the sense that congestion was assumed to be independent of the time of the day (see e.g. Sheffi (1981)). The static models were developed in different directions (heterogeneity, multiple types of vehicles, elastic demand, stochastic equilibrium ...). The treatment of congestion remained rather inappropriate because somewhat ad hoc peak and off-peak periods were introduced as a surrogate to take into account the time dependent nature of congestion. In reality, the choice between peak and off-peak must be endogenous as it depends on the level of congestion. This is also true with several policies, road pricing and change of the peak period lengths for instance, which have to be determined endogenously. One may assume cross-elasticities between the periods, but these elasticities should not be be constant.

Intermediary models have been proposed by Nesterov and de Palma (2003) for a stable dynamic approach between the static and dynamic cases. These models are popular in operations research since they can be formulated through some convex problem. In the present work, we will focus on bottleneck models whose construction is fully dynamic.

By contrast, in that dynamic modelling, congestion depends endogenously on the time of the day. Such models extend the dynamic assignment models considered for problems of route

\footnotetext{
${ }^{1}$ Corresponding author. CREST, Bâtiment Laplace, 61 avenue du Président Wilson, F-94230 Cachan, France. E-mail address: andre.depalma@ens-cachan.fr

${ }^{2}$ Département de Mathématique, Campus de la Plaine C.P. 210, Boulevard du Triomphe, B-1050 Bruxelles, Belgique. Email address: clefevre@ulb.ac.be
} 
choice. The initial dynamic congestion model is due to the economist W.S. Vickrey (1969). In this deterministic model, there is an origin, a destination and a single bottleneck of finite capacity. The cost function for the driver is the sum of the travel time and the schedule delay cost (i.e. the cost due to an early or late arrival); it is piece-wise linear.

Variants of this dynamic model were discovered, independently, by Moshe Ben-Akiva, Nicolas Litinas, Pavlos Kanaroglou and the two authors of this paper. The reader is referred to de Palma, Ben-Akiva, Lefèvre and Litinas (1983), Ben-Akiva, de Palma and Kanaroglou (1985, 1986a, 1986b) and de Palma, Lefèvre and Ben-Akiva (1987). These authors generalized the deterministic model à la Vickery in two ways. First, the demand side is represented by a discrete probabilistic model which is obtained from a continuous logit choice model. Second, the supply side is an extended bottleneck model inspired by Robert Herman, the pioneer of Transportation Science, who developed it with André during a visit at Austin, Texas.

In static bottleneck models, a major decision variable that was missing is the departure time choice. This variable is encapsulated in the fully dynamic models. The first computational model was developed with Pavlos who was then with André at McMaster University. A computer program was developed to compute a stochastic equilibrium in a small network. It was based on a day-to-day adjustment process (an iteration corresponds to a day). At the beginning of a day, the driver combines the experienced travel time with the expected travel time. At the end of the day, the driver has experienced another travel time. This allows the driver to revise its expectation of travel time for the next day according to a moving average process. That iteration procedure converges in general after about thirty steps.

This line of reseach work was continued and amplified in many papers. The early work in the economic literature is a seminal paper by Arnott, de Palma and Lindsey (1993). For a review, see e.g. de Palma and Fosgerau (2011). The literature on dynamic bottleneck models is now rich and diversified. In particular, a large scale dynamic model, METROPOLIS, was used in many cities all around the word; see de Palma and Marchal (1996) and de Palma, Marchal and Nesterov (1997). Recently, the driver simulation models DynaMit and MATSim were developed in a similar purpose by other researchers.

A first goal of the present paper is to provide both deterministic and stochastic versions of this dynamic setting. There is an enormous literature relying on a stochastic approach but it often neglects to describe endogenously the arrival rates. Besides that reason, we want to consider a stochastic bottleneck model because so far, there exists no simple day-to-day adjustment process which always leads to stable equilibria. Probabilistic birth-and-death master equations provide us with another angle of view to stability since they usually yield a stationarity distribution. As we know, a deterministic macroscopic stable solution is not necessarily the most likely outcome to the corresponding stochastic process. The reader is referred e.g. to the discussion made in de Palma and Lefèvre (1983). Let us also mention that stochasticity may play other roles in the bottleneck models. This is the case when considering stochastic capacities, as in Xiao, Huang and Liu (2015), or stochastic travel times, as in Xiao, Coulombel and de Palma (2017).

A second goal of this paper is to introduce in the model a combination of small and large transport agents. A driver is considered as small when its departure time has no influence 
on the aggregate level of congestion. We consider a mix of small drivers and of a large fleet of vehicles which have similar sizes (the case of variable sizes could be discussed similarly). The departure time choices in the fleet are assumed to be taken by a single manager whose objective is to minimize his various costs by taking into account the consequences of his choices. This decision process is assumed here to occur continuously in time, so that a small number of vehicles of the fleet can be reallocated at each instant. The fleet is large to guarantee that the cost per vehicle is of the same order of magnitude as the externality. The case where the manager decides periodically, at discrete times, is left for a future research.

The structure of the paper is as follows. In Section 2, we construct the basic deterministic model: the supply side is the bottleneck model such as introduced by Vickrey (1969), while the demand side is a logit discrete choice model. In Section 3, we examine the stochastic version of this model by resorting to birth-and-death master equations (see e.g. Ross (1996)). In Section 4 , we introduce a deterministic model which combines small and large agents. We compute the equilibrium state by showing that the individual and fleet vehicles behave rather differently. In Section 5, we formulate this model in a stochastic framework, with a special attention to the choice problem between two departure times.

\section{$2 \quad$ Starting deterministic model}

We first present a deterministic dynamic model for congestion. In this model, we adopt the traditional assumption that all the drivers have the same importance for the traffic. We also assume that each driver is arbitrarily small so that the equilibrium concept is of Nash with a continuum of users.

\subsection{Definitions and notation}

Let us introduce the key parameters of the model:

$t$, the departure time $(t \in[0, T]$, continuous or not),

$\omega$, the iteration (day, $\omega=0, \ldots, \Omega$ ),

$r_{\omega}(t)$, the number of drivers departing from the origin at time $t$ at iteration $\omega$,

$R_{\omega}(t)$, the cumulated inflow at time $t$ at iteration $\omega$, i.e. $R_{\omega}(t)=\int_{0}^{t} r_{\omega}(u) d u$,

$N$, the total number of drivers $\left(\int_{0}^{T} r_{\omega}(t) d t=N\right)$,

$s$, the capacity of the bottleneck (number of vehicles per hour),

$Q_{\omega}(t)$, the number of users in the queue at time $t$ at iteration $\omega$,

$t t_{\omega}(t)=Q_{\omega}(t) / s$, the waiting time of a driver at the bottleneck at time $t$ at iteration $\omega$.

Without loss of generality, the free-flow travel time is assumed to be 0 . Let $\tau_{\omega}(t)$ denote the last time before $t$ when congestion started (provided it occurs) at iteration $\omega$. So,

$$
\tau_{\omega}(t)=\sup \left\{u \leq t: Q_{\omega}(u)>0\right\} .
$$

When there is congestion, the queue length is then given by

$$
Q_{\omega}(t)=\int_{\tau_{\omega}(t)}^{t} r_{\omega}(u) d u-s\left[t-\tau_{\omega}(t)\right]
$$


The cost for a driver departing at time $t$ at iteration $\omega$ is defined by

$$
C_{\omega}(t)=\alpha t t_{\omega}(t)+\beta\left[t^{*}-t_{\omega}^{a}(t)\right]^{+}+\gamma\left[t_{\omega}^{a}(t)-t^{*}\right]^{+},
$$

in which

$t^{*}$ is the desired time of destination,

$t_{\omega}^{a}(t)$ is the arrival time of a driver departing at time $t$ at iteration $\omega$, i.e. $t_{\omega}^{a}(t)=t+t t_{\omega}(t)$.

\subsection{Departure pattern}

Denote by $P_{\omega}\left(t \mid t^{\prime}\right)$ the transition probability from departure choice $t^{\prime}$ at iteration $\omega-1$ to departure time $t$ at iteration $\omega$. Note that $t$ may be larger or smaller than $t^{\prime}$. That probability is assumed to be of the form

$$
P_{\omega}\left(t \mid t^{\prime}\right)=\frac{h_{\omega}\left(t \mid t^{\prime}\right)}{H_{\omega}\left(t^{\prime}\right)}
$$

where $H_{\omega}\left(t^{\prime}\right)=\int_{0}^{T} h_{\omega}\left(t \mid t^{\prime}\right) d t$ is the aggregator at iteration $\omega$ and yields, of course,

$$
\int_{0}^{T} P_{\omega}\left(t \mid t^{\prime}\right) d t=1
$$

Then, the number of drivers departing at time $t$ at iteration $\omega$ is given by

$$
r_{\omega}(t)=\int_{0}^{T} P_{\omega}\left(t \mid t^{\prime}\right) r_{\omega-1}\left(t^{\prime}\right) d t^{\prime}
$$

The drivers are assumed to be small enough so that they may consider that their departure time decisions do not affect the value of the aggregator. That is, during each period, the value of the aggregator is constant from the driver point of view. This is an assumption similar to the monopolistic competition (see Dixit and Stiglitz (1977)).

\subsection{Logit probabilities}

In (2.3), the function $h_{\omega}\left(t \mid t^{\prime}\right)$ depends on the cost incurred at iteration $\omega-1$ at time $t^{\prime}$ and on the cost incurred at iteration $\omega$ at time $t$.

We consider a logit specification for the transition probabilities. The logit model is a classical tool in Transportation (McFadden (2001)) and Industrial Organization (Anderson, de Palma and Thisse (1992)). Recently, it was also used in the study of Big Data, especially for sentiment analysis (Yessenalina and Cardie (2011)). Specifically, we choose

$$
h_{\omega}\left(t \mid t^{\prime}\right)=e^{-\left[C_{\omega}(t)-C_{\omega-1}\left(t^{\prime}\right)\right]} .
$$

In this case, (2.3) is given by the continuous logit formula

$$
P_{\omega}\left(t \mid t^{\prime}\right)=\frac{e^{-C_{\omega}(t)}}{\int_{0}^{T} e^{-C_{\omega}(u) d u}} \equiv P_{\omega}(t)
$$


It is worth recalling that when the error terms in the logit are of zero variance, this model reduces to the deterministic case. From (2.5), we observe that the transition probability becomes here independent of $t^{\prime}$, which makes easier the model analysis. Using (2.4), we then obtain that

$$
r_{\omega}(t)=\int_{0}^{T} P_{\omega}(t) r_{\omega-1}\left(t^{\prime}\right) d t^{\prime} \equiv N P_{\omega}(t) .
$$

Let us check that this system has $r_{\omega}(t)$ as a fixed point. Indeed, from (2.5) and (2.6),

$$
r_{\omega}(t)=N \frac{e^{-C_{\omega}(t)}}{\int_{0}^{T} e^{-C_{\omega}(u) d u}} .
$$

We postulate that the equilibrium solution has the following intuitive pattern over time: first, departures occur without congestion early (arrival before $t^{*}$ ); then, departures occur with congestion early; next, departures occur with congestion late; finally, departures occur without congestion late. Let $t_{\omega}^{i}$ be the first early departure with congestion and let $t_{\omega}^{f}$ be the first late departure without congestion. For early arrivals, we have

$$
C_{\omega}^{E}(t)=(\alpha-\beta) t t_{\omega}(t)+\beta\left(t^{*}-t\right), \quad t<\hat{t}_{\omega}
$$

where $t_{\omega}^{a}\left(\hat{t}_{\omega}\right)=t^{*}$. Likewise, for late arrivals,

$$
C_{\omega}^{L}(t)=(\alpha+\gamma) t t_{\omega}(t)+\gamma\left(t-t^{*}\right), \quad t>\hat{t}_{\omega} .
$$

When there is no congestion, the travel time is constant (normalized to 0 with wlog). When there is congestion, the travel time is given by

$$
t t_{\omega}(t)=\left[\int_{t_{\omega}^{i}}^{t} r_{\omega}(u) d u-s\left(t-t_{\omega}^{i}\right)\right]_{s}, \quad t_{i} \leq t \leq t_{\omega}^{f} .
$$

So, the choice probabilities $P_{\omega}(t)$ depend on the cost function which depends on the travel time, which itself depends on the departure time schedule $\left\{r_{\omega}(u)\right\}$. This shows that the departure rate is a solution of the fixed point problem.

We have assumed above that each driver at iteration $\omega$ selects its departure time at the beginning of the iteration with full knowledge of travel time for each departure time and solves the fixed point accordingly. Alternatively, we assume that a driver has not a perfect foresight and builds at the beginning of iteration $\omega$ an estimate of the travel times for the whole period. The estimation made for period $\omega$ depends on the estimation made for period $\omega-1$ and on the observed travel times during period $\omega-1$, i.e.

$$
t t_{\omega}^{a p}(t)=g\left[t t_{\omega-1}^{a p}(t), t t_{\omega-1}^{o b}(t)\right] .
$$

In the general case, the transition probabilities might depend explicitly on the previous choice $t^{\prime}$ at iteration $\omega-1$.

Estimation of departure time choices is standard using an ordered probit model (see e.g. Small (1982)). A logit model for the departure time choices is possible too, on the basis of stated or revealed data. In the next section, we propose a stochastic approach which is more natural to describe the departure time process. It has not been considered so far. 


\section{A stochastic framework}

This time, we assume that the departure times are discrete $(t=0, \ldots, T)$ and the iteration process is Markovian in continuous time $\left(\omega \in \mathbb{R}_{+}\right)$. This provides us with a framework which is standard and convenient in probability theory (see e.g. Ross (1996)). Note that considering a continuous time $\omega$ yields an approximation to the daily decision process which is reasonable in the long term.

Let $X_{\omega}(t)$ be the number of drivers departing at time $t$ at iteration $\omega$. We want to derive the joint distribution of the vector $\mathbf{X}_{\omega}=\left\{X_{\omega}(t), t=0, \ldots, T\right\}$. Let $p_{\omega}(\mathbf{x})=P\left(\mathbf{X}_{\omega}=\mathbf{x}\right)$, with $p_{0}(\mathbf{x})$ supposed to be fixed.

Using the Markovian property, we get the master equation

$$
\begin{array}{r}
\frac{d p_{\omega}(\mathbf{x})}{d \omega}=\sum_{j \mid x_{j} \geq 1} \sum_{i \neq j \mid x_{i} \leq N-1}\left(x_{i}+1\right) \\
p_{\omega}\left(\mathbf{x}+\mathbf{e}_{i}-\mathbf{e}_{j}\right) g_{\omega}^{j \mid i}\left(\mathbf{x}+\mathbf{e}_{i}-\mathbf{e}_{j}\right) \\
-p_{\omega}(\mathbf{x}) \sum_{j \mid x_{j} \geq 1} \sum_{i \neq j \mid x_{i} \leq N-1} x_{i} g_{\omega}^{j \mid i}(\mathbf{x}),
\end{array}
$$

where $\mathbf{e}_{i}$ is the vector with 1 at the $i$-th position and 0 elsewhere, and where $g_{\omega}^{j \mid i}(\mathbf{x})$ denotes the transition rate from departure time $i$ to departure time $j$ at iteration $\omega$ when the present state is $\mathbf{x}$. This rate is a function of the form

$$
g_{\omega}^{j \mid i}(\mathbf{x})=f\left[C_{\omega}(i), C_{\omega}(j)\right],
$$

where the cost $C_{\omega}(t)$ is defined as before by (2.2) and depends on $t$ and $\left\{X_{\omega}(u), u=0, \ldots, t\right\}$. The latter property comes from the fact that the cost at time $t$ depends on travel times at time $t$, which depend themselves on departures before time $t$. Thus, $g_{\omega}^{j \mid i}(\mathbf{x})$ depends in fact on $\mathbf{x}_{i \vee j}=\left(x_{1}, \ldots, x_{i \vee j}\right)$ only. In (3.2), the function $f(x, y)$ is expected to be increasing in $x$ and decreasing in $y$.

The process $\left\{\mathbf{X}_{\omega}, \omega \in \mathbb{R}_{+}\right\}$corresponds to a multi-dimensional birth-and-death process on $[0, N]^{T+1}$. As the state space is finite, the equations (3.1) admit, for all $\omega$, a unique solution $p_{\omega}(\mathbf{x})$ under certain conditions on the transition rates. Moreover, this solution can then converge towards a stationary distribution $p(\mathbf{x})$. Further details can be found e.g. in Zeifman (1995).

Note that a single driver might be considered as small with respect to the total mass of drivers. In such a case, it can neglect the impact of its decision on the travel pattern. Mathematically, this means that in (3.1), the transition rate $g_{\omega}^{j \mid i}\left(\mathbf{x}+\mathbf{e}_{i}-\mathbf{e}_{j}\right)$ can be aproximated by $g_{\omega}^{j \mid i}(\mathbf{x})$.

\section{Small and large agents: a deterministic setting}

Let us pass to a more general model where the drivers can have a variable importance. In this section, we deal with the deterministic approach, directly at the stationary state (so that the index $\omega$ becomes superfluous). 
There are now a number $N$ of individual drivers and a large fleet of $M$ vehicles that is managed by a single decision maker. The manager of the fleet takes into account the congestioninduced by its decision. The choice process is made in two stages. At the first step, all the individual drivers may choose their departure time according to the method described in Section 3. At the next step, the manager decides to reallocate the vehicles of its fleet by estimating the generated congestion. The fleet is large so that the cost per vehicle is approximately of the same level as the externality (see below).

\subsection{Binary choice}

Let us first discuss the case where there are only two possible departure times 1 and 2 . The cost per vehicle in the fleet are given at time 1 by $C_{1}^{A}=h_{1}\left(X_{1}+Y_{1}\right)$, and at time 2 by $C_{2}^{A}=h_{2}\left(\theta X_{1}+\theta Y_{1}+X_{2}+Y_{2}\right)$ where $0<\theta<1$. The functions $h_{1}$ and $h_{2}$ are increasing since the costs increase with the number of vehicles. The parameter $\theta$ translates the fact that the vehicles departing at time 1 are linearly less costly for those departing later at time 2 . The cost of the whole fleet of vehicles is thus equal to $C^{A}=Y_{1} C_{1}^{A}+Y_{2} C_{2}^{A}$.

Suppose now that $\delta_{Y}$ vehicles from the fleet change their previous choice and decide to depart at time 2 instead of time 1 . The direct cost generated by these $\delta_{Y}$ vehicles is

$$
\begin{aligned}
\Delta^{D}\left(C^{A}\right) & =\delta_{Y}\left[-h_{1}\left(X_{1}+Y_{1}-\delta_{Y}\right)+h_{2}\left(\theta X_{1}+\theta Y_{1}-\theta \delta_{Y}+X_{2}+Y_{2}+\delta_{Y}\right)\right] \\
& =\delta_{Y}\left[-h_{1}\left(X_{1}+Y_{1}\right)+h_{2}\left(\theta X_{1}+\theta Y_{1}+X_{2}+Y_{2}\right)\right]+O\left(\delta_{Y}^{2}\right) .
\end{aligned}
$$

Therefore,

$$
\Delta^{D}\left(C^{A}\right)=\delta_{Y}\left[-C_{1}^{A}+C_{2}^{A}+O\left(\delta_{Y}\right)\right]
$$

and as $\delta_{Y}$ is small with respect to the costs $C_{i}^{A}, i=1,2$,

$$
\Delta^{D}\left(C^{A}\right) \approx \delta_{Y}\left(-C_{1}^{A}+C_{2}^{A}\right) .
$$

Let us examine the impact of the change made by the $\delta_{Y}$ vehicles on the other vehicles of the fleet. Using as before first-order approximations, we obtain for the generated indirect cost

$$
\begin{aligned}
\Delta^{I}\left(C^{A}\right)= & \left(Y_{1}-\delta_{Y}\right)\left[h_{1}\left(X_{1}+Y_{1}-\delta_{Y}\right)-h_{1}\left(X_{1}+Y_{1}\right)\right]+ \\
& +\left(Y_{2}+\delta_{y}\right)\left[h_{2}\left(\theta X_{1}+\theta Y_{1}-\theta \delta_{Y}+X_{2}+Y_{2}+\delta_{Y}\right)-h_{2}\left(\theta X_{1}+\theta Y_{1}+X_{2}+Y_{2}\right)\right] \\
= & \delta_{Y}\left[-Y_{1} h_{1}^{\prime}\left(X_{1}+Y_{1}\right)+Y_{2}(1-\theta) h_{2}^{\prime}\left(\theta X_{1}+\theta Y_{1}+X_{2}+Y_{2}\right)\right]+O\left(\delta_{Y}^{2}\right),
\end{aligned}
$$

where $f^{\prime}$ denotes the derivative of a function $f$. Therefore,

$$
\Delta^{I}\left(C^{A}\right) \approx \delta_{Y}\left[Y_{1} C_{1}^{\prime A}+Y_{2}(1-\theta) C_{2}^{A}\right] .
$$

The total impact due to the $\delta_{Y}$ changes is $\Delta\left(C^{A}\right)=\Delta^{D}\left(C^{A}\right)+\Delta^{I}\left(C^{A}\right)$. From (4.1) and (4.2), we then get

$$
\Delta\left(C^{A}\right) \approx \delta_{Y}\left\{C_{2}^{A}-C_{1}^{A}+\left[Y_{2}(1-\theta) C_{2}^{A}-Y_{1} C_{1}^{A}\right]\right\}
$$


We are in a position to make precise the assumption that the fleet is large enough. By this, we mean that

Assumption: $C_{i}^{A}$ is of the same order of magnitude as $Y_{i} C_{i}^{\prime A}, \quad i=1,2$.

Let us recall that the externality, denoted by $e_{i}^{A}$, is the difference between the marginal social cost, $\left(Y_{i} C_{i}^{A}\right)^{\prime}=Y_{i} C_{i}^{\prime A}+C_{i}^{A}$, and the individual cost $C_{i}^{A}$; so, $e_{i}^{A}=Y_{i} C_{i}^{\prime A}$. Therefore, the assumption above states that the cost $C_{i}^{A}$ per vehicle in the fleet is of the same order of magnitude as the externality $e_{i}^{A}$.

\subsection{Equilibrium}

The choice process begins with the individual drivers. They select between the departure times 1 and 2 and at the equilibrium, the associated costs are equal. We consider a Nash equilibrium, so that each driver considers that the decision of the others is fixed. The costs per individual are given at time 1 by $C_{1}^{I}=g_{1}\left(X_{1}+Y_{1}\right)$, and at time 2 by $C_{2}^{I}=g_{2}\left(\theta X_{1}+\theta Y_{1}+X_{2}+Y_{2}\right)$ where the parameter $\theta$ is defined as for the fleet vehicles. The functions $g_{1}$ and $g_{2}$ are again increasing.

At equilibrium, we have

$$
C_{1}^{I}=g_{1}\left(X_{1}+Y_{1}\right)=C_{2}^{I}=g_{2}\left(\theta X_{1}+\theta Y_{1}+X_{2}+Y_{2}\right)
$$

Since $X_{1}+X_{2}=N$, (4.4) becomes

$$
g_{1}\left(X_{1}+Y_{1}\right)=g_{2}\left(\theta X_{1}+\theta Y_{1}+N-X_{1}+M-Y_{1}\right) .
$$

Let us assume that there is an interior solution. It is unique since the left-hand side is increasing in $X_{1}$, while the right-hand side is decreasing in $X_{1}$. That solution is denoted by $X_{1}^{b r}=$ $\gamma\left(Y_{1} ; N, M\right)$, with $d X_{1}^{b r} / d Y_{1}<0$.

Then, it is the turn of the drivers in the fleet. The fleet manager minimizes the total cost of the fleet taking constant the decisions of the individual drivers. At the optimum, it is necessary and sufficient that any change has no impact on the cost. This means that $\Delta\left(C^{A}\right)=0$, i.e. from (4.3),

$$
\delta_{Y}\left(-C_{1}^{A}+C_{2}^{A}\right)+\delta_{Y}\left[-Y_{1} C_{1}^{\prime A}+Y_{2}(1-\theta) C_{2}^{\prime A}\right]=0 .
$$

After simplification by $\delta_{Y}$, we get

$$
C_{1}^{A}+Y_{1} C_{1}^{A}=C_{2}^{A}+Y_{2}(1-\theta) C_{2}^{\prime A} .
$$

Equation (4.6) is equivalent to

$$
\begin{aligned}
h_{1}\left(X_{1}+Y_{1}\right)+Y_{1} h_{1}^{\prime}\left(X_{1}+Y_{1}\right)= & h_{2}\left(\theta X_{1}+\theta Y_{1}+N-X_{1}+M-Y_{1}\right) \\
& +Y_{2}(1-\theta) h_{2}^{\prime}\left(\theta X_{1}+\theta Y_{1}+N-X_{1}+M-Y_{1}\right) .
\end{aligned}
$$


As before, assume that there is an interior solution. It is unique here too and denoted by $Y_{1}^{b r}=\mu\left(X_{1} ; N, M\right)$, with $d Y_{1}^{b r} / d X_{1}<0$.

From (4.5) and (4.7), a Nash equilibrium state $\left(X_{1}^{*}, Y_{1}^{*}\right)$ will satisfy the following system

$$
\left\{\begin{array}{l}
X_{1}^{*}=\gamma\left(Y_{1}^{*} ; N, M\right) \\
Y_{1}^{*}=\mu\left(X_{1}^{*} ; N, M\right)
\end{array}\right.
$$

Of course, $X_{2}^{*}=N-X_{1}^{*}$ and $Y_{2}^{*}=M-Y_{1}^{*}$. Let us pursue with a more analytical formulation.

Linear costs. We now assume that the costs are linear with, for instance, $g_{1}=g_{2}=h_{1}=h_{2}$ the identity fuction. Then, the individual condition (4.5) becomes

$$
\left(X_{1}+Y_{1}\right)=\left(\theta X_{1}+\theta Y_{1}+X_{2}+Y_{2}\right)
$$

which can be rewritten as

$$
X_{1}(2-\theta)+Y_{1}(2-\theta)=M+N .
$$

Moreover, the fleet condition (4.7) becomes

$$
\left(X_{1}+Y_{1}\right)+Y_{1}=\theta X_{1}+\theta Y_{1}+N-X_{1}+M-Y_{1}+\left(M-Y_{1}\right)(1-\theta),
$$

which reduces to

$$
X_{1}(2-\theta)+Y_{1}(4-2 \theta)=N+M(2-\theta) .
$$

Combining (4.8) and (4.9), we obtain a system of two linear equations with thwo unknowns. Its solution is found to be

$$
\left\{\begin{array}{l}
X_{1}^{*}=(N+\theta) /(2-\theta), \text { and thus } X_{2}^{*}=[N(1-\theta)-\theta] /(2-\theta) \\
Y_{1}^{*}=(M-\theta) /(2-\theta), \text { and thus } Y_{2}^{*}=[M(1-\theta)+\theta] /(2-\theta)
\end{array}\right.
$$

Note that $0<Y_{1}^{*}, Y_{2}^{*}<M$, while $0<X_{1}^{*}, X_{2}^{*}<N$ if $\theta<N /(N+1)$, which will be often satisfied in practice.

\subsection{Extension}

Let us now examine a more general model with $K$ possible departure times . The vehicles departed before any time $j, 1 \leq j \leq K$, influence the costs for the fleet vehicles and the individual drivers through the polynomial function

$$
\sum_{r=0}^{j-1} \theta^{j-r} x_{r} .
$$

A similar reasoning will allow us to obtain the equilibrium state by recursion. For instance, take $K=3$. We start by considering the first two departure times. By the previous method, we obtain a solution $\left\{X_{1}^{*}, Y_{1}^{*}, X_{2}^{*}, Y_{2}^{*}\right\}$. For the individual drivers, the common cost, denoted by $C^{I}$, satisfies (4.4), i.e.

$$
g_{1}\left(X_{1}^{*}+Y_{1}^{*}\right)=C^{I}=g_{2}\left(\theta X_{1}^{*}+\theta Y_{1}^{*}+X_{2}^{*}+Y_{2}^{*}\right) .
$$


At equilibrium, the costs for the individuals are the same for the $K$ times, which yields

$$
g_{3}\left(\theta^{2} X_{1}^{*}+\theta^{2} Y_{1}^{*}+\theta X_{2}^{*}+\theta Y_{2}^{*}+X_{3}+Y_{3}\right)=C^{I}
$$

For the vehicles in the fleet, the common cost, denoted by $M S C^{A}$ (marginal social cost), is given by

$$
M S C^{A}=h_{1}\left(X_{1}^{*}+Y_{1}^{*}\right)+Y_{1}^{*} h_{1}^{\prime}\left(X_{1}^{*}+Y_{1}^{*}\right),
$$

and from (4.7), we also have

$$
M S C^{A}=h_{2}\left(\theta X_{1}^{*}+\theta Y_{1}^{*}+X_{2}^{*}+Y_{2}^{*}\right)+Y_{2}^{*}(1-\theta) h_{2}^{\prime}\left(\theta X_{1}^{*}+\theta Y_{1}^{*}+X_{2}^{*}+Y_{2}^{*}\right) .
$$

Here again, at equilibrium, the marginal social costs are identical for the $K$ times, so that

$$
\begin{gathered}
h_{3}\left(\theta^{2} X_{1}^{*}+\theta^{2} Y_{1}^{*}+\theta X_{2}^{*}+\theta Y_{2}^{*}+X_{3}+Y_{3}\right)+Y_{3} h_{3}^{\prime}\left(\theta^{2} X_{1}^{*}+\theta^{2} Y_{1}^{*}+\theta X_{2}^{*}+\theta Y_{2}^{*}+X_{3}+Y_{3}\right) \\
=M S C^{A} .
\end{gathered}
$$

The system (4.10) and (4.11) will have a unique solution $X_{3}^{*}$ and $Y_{3}^{*}$, under some regulatory conditions. The procedure can then continue recursively.

\section{$5 \quad$ Small and large agents: a stochastic setting}

This time, we will use a Markovian model in continuous-time (see e.g. Ross (1996)) to describe the decision process of the individual drivers and the fleet manager.

\subsection{Binary choice}

We start again by considering two possible departure times 1 and 2 . Let $\left(X_{\omega}, Y_{\omega}\right)$ be the numbers of individual drivers and vehicles in the fleet that choose to depart at time 2 at iteration $\omega$. Note that the number of departures at time 1 is $\left(N-X_{\omega}, M-Y_{\omega}\right)$. We are interested in the joint distribution of the vector $\left(X_{\omega}, Y_{\omega}\right)$. Let $p_{\omega}(x, y)=P(X=x, Y=y)$, with $p_{0}(x, y)$ supposed to be fixed .

From the Markovian assumption, we obtain the master equations

$$
\begin{aligned}
\frac{d p_{\omega}(x, y)}{d \omega}= & p_{\omega}(x, y+1) f_{\omega}^{2 \mid 1}(x, y+1)+p_{\omega}(x, y-1) f^{1 \mid 2}(x, y-1) \\
& +p_{\omega}(x+1, y)(x+1) g_{\omega}^{2 \mid 1}(x+1, y)+p_{\omega}(x-1, y)(N-x+1) g_{\omega}^{1 \mid 2}(x-1, y) \\
& -p_{\omega}(x, y)\left[f_{\omega}^{2 \mid 1}(x, y)+f^{1 \mid 2}(x, y)+x g_{\omega}^{2 \mid 1}(x, y)+(N-x) g_{\omega}^{1 \mid 2}(x, y)\right],
\end{aligned}
$$

where, for instance, $f_{\omega}^{2 \mid 1}(x, y)$ (resp. $g_{\omega}^{2 \mid 1}(x, y)$ ) is the fleet transition rate (resp. individual transition rate) from departure time 1 to departure time 2 at iteration $\omega$ when the state is $(x, y)$ at iteration $\omega-1$. The transition rates at state $(x, y)$ equal 0 when $x$ is outside $[0, N]$ or $y$ is outside $[0, M]$. 
The transition rates can be constructed by means of a logit model. Let us omit the argument $\omega$ to simplify the notation. For the fleet, arguing as for (4.2), this leads to

$$
f^{2 \mid 1}(x, y)=\lambda \frac{e^{-\left[C_{2}^{A}(x, y)+y(1-\theta) C_{2}^{A \prime}(x, y)\right]}}{e^{-\left[C_{1}^{A}(x, y)+(M-y) C_{1}^{A \prime}(x, y)\right]}+e^{-\left[C_{2}^{A}(x, y)+y(1-\theta) C_{2}^{A \prime}(x, y)\right]}},
$$

where $\lambda$ is the decision-making rate of the manager and $C_{i}^{A}(x, y), i=1,2$, is the cost of a vehicle in the fleet departing at time $i$. For the individual drivers, we have

$$
g^{2 \mid 1}(x, y)=\mu \frac{e^{-C_{2}^{I}(x, y)}}{e^{-C_{1}^{I}(x, y)}+e^{-C_{2}^{I}(x, y)}},
$$

where $\mu$ is the individual decision-making rate and $C_{i}^{I}(x, y), i=1,2$, is the cost of a driver departing at time $i$.

The process $\left\{\left(X_{\omega}, Y_{\omega}\right), \omega \in \mathbb{R}_{+}\right\}$corresponds to a bivariate birth-and-death process on $[0, N] \times[0, M]$. The associated equations (5.1) admit a unique solution $p_{\omega}(x, y)$ for all $\omega$ and a stationary solution $p(x, y)$, under some regularity conditions on the rates.

\subsection{General case}

Let $X_{\omega}(t)$ be the number of individual drivers departing at time $t$ at iteration $\omega$, and let $Y_{\omega}(t)$ be the number of vehicles of the fleet departing at time $t$ at iteration $\omega$. We want to derive the joint distribution of the vector $\left(\mathbf{X}_{\omega}, \mathbf{Y}_{\omega}\right)=\left\{\left[X_{\omega}(t), t=0, \ldots, T\right],\left[Y_{\omega}(t), t=0, \ldots, T\right]\right\}$. Let $p_{\omega}(\mathbf{x}, \mathbf{y})=P\left(\mathbf{X}_{\omega}=\mathbf{x}, \mathbf{Y}_{\omega}=\mathbf{y}\right)$, with $p_{0}(\mathbf{x}, \mathbf{y})$ supposed to be fixed.

In this case, the master equations become

$$
\begin{aligned}
\frac{d p_{\omega}(\mathbf{x}, \mathbf{y})}{d \omega}= & \sum_{\mathbf{z} \neq \mathbf{y}} p_{\omega}(\mathbf{x}, \mathbf{z}) f_{\omega}^{\mathbf{y} \mid \mathbf{z}}(\mathbf{x}, \mathbf{z}) \\
& +\sum_{j \mid x_{j} \geq 1} \sum_{i \neq j \mid x_{i} \leq N-1}\left(x_{i}+1\right) p_{\omega}\left(\mathbf{x}+\mathbf{e}_{i}-\mathbf{e}_{j}, \mathbf{y}\right) g_{\omega}^{j \mid i}\left(\mathbf{x}+\mathbf{e}_{i}-\mathbf{e}_{j}, \mathbf{y}\right) \\
& -p_{\omega}(\mathbf{x}, \mathbf{y})\left[\sum_{\mathbf{z} \neq \mathbf{y}} f^{\mathbf{y} \mid \mathbf{z}}(\mathbf{x}, \mathbf{y})+\sum_{j \mid x_{j} \geq 1} \sum_{i \neq j \mid x_{i} \leq N-1} x_{i} g_{\omega}^{j \mid i}(\mathbf{x}, \mathbf{y})\right],
\end{aligned}
$$

using the same notation as for (5.1). To the best of our knowledge, such a birth-and-death process is a new framework for the congestion problems. It would be interesting to simulate this stochastic process, assuming a smooth behavior for the transition rates.

\section{Conclusions}

We have developed two main ideas in this work. The first one is the use of a stochastic approach to tackle dynamic transportation bottleneck models. Equilibrium solutions can be determined in various situations but very little is known concerning the adjustment process and 
the stability. We believe that the problem is more amenable to numerical simulation when it is formulated in a truly stochastic environment (i.e., not only with discrete choice models but with birth-and-death master equations).

Secondly, we have taken into account the possible presence of a fleet of vehicles in the model. The technology of tomorrow suggests that fleets of vehicles will become more frequent in the real world (e.g., vehicles on demand that are centrally managed ...). These issues were rarely addressed in the literature. We have considered here a tractable way to combine individual vehicles and a fleet of vehicles which are centrally managed.

Such a model can raise questions that are mathematically difficult. As shown by Silva, Lindsey, de Palma and van den Berg (2017), a combination of small and large vehicles can lead to some non-equilibrium in the bottleneck problem. This situation cannot be easily avoided and is very unfortunate. The stochastic approach is more realistic and flexible, and it is expected to provide us with an equilibrium solution even in such a case.

The relation between stochastic and deterministic models is an important but difficult topic (see e.g. the book of Ethier and Kurtz (2005)). In our context, two relevant questions are the links between the local extrema of (stochastic) stationary distributions and the (deterministic) equilibrium states, and the existence of a solution in a stochastic model while there is no solution in the deterministic case.

At this point, we were mainly concerned with the modelling problems. Simulation of these processes and stationarity properties are topics that will be the object of further research.

\section{Acknowledgements}

We thank the referee and the editor for very helpful and constructive comments. The research of André de Palma was supported by the ANR Project Elitisme from the French Agence Nationale de la Recherche. The research of Claude Lefèvre was supported by the ARC Project IAPAS from the Fédération Wallonie-Bruxelles. This work has benefited from many discussions with Moshe and Pavlos during several visits in the past at the Massachusetts Institute of Technology (MIT).

\section{References}

Anderson, S.P., de Palma, A. and Thisse, J.-F. (1992). Discrete Choice Theory of Product Differentiation. MIT Press, Cambridge.

Arnott, R., de Palma, A., and Lindsey, R. (1993). A structural model of peak-period congestion: A traffic bottleneck with elastic demand. American Economic Review 83, 161-179.

Ben-Akiva, M., de Palma, A. and Kanaroglou, P. (1986a). Dynamic model of peak period traffic congestion with elastic arrival rates. Transportation Science 20, 164-181.

Ben-Akiva, M., de Palma, A. and Kanaroglou, P. (1986b). Effects of capacity constraints on peak period congestion. Transportation Research Record 1085, 16-26. 
Ben-Akiva, M., de Palma, A., and Kanaroglou, P. (1987). Dynamic network equilibrium: Some comments. European Journal of Operational Research 30, 318-320.

de Palma, A. and Lefèvre, C. (1983). Individual decision-making in dynamic collective systems. Journal of Mathematical Sociology 9, 103-124.

de Palma, A., Ben-Akiva, M., Lefèvre, C. and Litinas, N. (1983). Stochastic equilibrium model of peak period traffic congestion. Transportation Science 17, 430-453.

de Palma, A., Lefèvre, C. and Ben-Akiva, M. (1987). A dynamic model of peak period flows and delays in a corridor. Computers and Mathematics with Applications 14, 201-223.

de Palma, A. and Marchal, F. (1996). METROPOLIS: Un outil de simulation du trafic urbain. Revue Transport 378, 304-315.

de Palma, A., F. Marchal, F. and Nesterov, Y. (1997). METROPOLIS: Modular system for dynamic traffic simulation. Transportation Research Record 1607, 178-184.

de Palma, A. and Fosgerau, M. (2011). Dynamic traffic modeling. In A Handbook of Transport Economics, A. de Palma, R. Lindsey, E. Quinet and R. Vickerman (eds), Edward Elgar, Cheltenham, 188-212.

Dixit, A.K. and Stiglitz, J.E. (1977). Monopolistic competition and optimum product diversity. American Economic Review 67, 297-308.

Ethier, S.N. and Kurtz, T.G. (2005). Markov Processes: Characterization and Convergence. Wiley, New York.

McFadden, D. (2001). Economic choices. American Economic Review 91, 351-378.

Nesterov, Y. and de Palma, A. (2003). Stationary dynamic solutions in congested transportation networks: Summary and perspectives. Networks and Spatial Economics 3, 371-395.

Ross, S.M. (1996). Stochastic Processes (2nd. edition). Wiley, New York.

Sheffi, Y. (1985). Urban Transportation Networks. Prentice-Hall, Englewood Cliffs.

Silva, H.E., Lindsey, R., de Palma, A. and van den Berg, V.A.C. (2017). On the existence and uniqueness of equilibrium in the bottleneck model with atomic users. Transportation Science 51, 863-881.

Small, K.A. (1982). The scheduling of consumer activities: Work trips. American Economic Review 72, 467-479.

Vickrey, W.S. (1969). Congestion theory and transport investment. American Economic Review 59, 251-260.

Xiao, L.-L., Huang, H.-J. and Liu, R. (2015). Congestion behavior and tolls in a bottleneck model with stochastic capacity. Transportation Science 49, 46-65.

Xiao, Y., Coulombel, N. and de Palma, A. (2017). The valuation of travel time reliability: does congestion matter? Transportation Research Part B: Methodological 97, 113-141.

Yessenalina, A. and Cardie, C. (2011). Compositional matrix-space models for sentiment analysis. In Proceedings of the Conference on Empirical Methods in Natural Language Processing, Association for Computational Linguistics, Stroudsburg, 172-182.

Zeifman, A.I. (1995). Upper and lower bounds on the rate of convergence for nonhomogeneous birth and death processes. Stochastic Processes and their Applications 59, 157-173. 